\title{
PERUMUMAN LEMMA SNAKE DAN LEMMA LIMA
}

\author{
Sripatmi $^{1}$, Yunita Septriana Anwar ${ }^{2}$ \\ ${ }^{1}$ Program Studi Pendidikan Matematika FKIP Universitas Mataram \\ ${ }^{2}$ Program Studi Pendidikan Matematika FKIP Universitas Muhammadiyah Mataram \\ Email: spatmi@gmail.com,na2_math@yahoo.com
}

\begin{abstract}
Abstrak. Barisan $U$-eksak merupakan perumuman dari barisan eksak yang diperkenalkan oleh Davvaz dan Parnian-Garamaleky. Dalam tulisan ini akan dikaji perumuman dari Lemma Snake dan Lemma Lima yang memanfaatkan sifat-sifat dari barisan $U$-eksak.
\end{abstract}

Kata Kunci : Barisan $U$-eksak, Lemma Snake, Lemma Lima

\begin{abstract}
U$-exact sequences was introduced by Davvaz dan Parnian-Garamaleky as a generalization of exact sequences. In this paper, we give some characterizations and properties of $U$-exact sequences. We use these result to find a generalization of Snake Lemma and Five Lemma.
\end{abstract}

Key words Kunci : $U$-exact sequences, Snake Lemma, Five Lemma

\section{PENDAHULUAN}

Diasumsikan $R$ adalah ring asosiatif dengan elemen identitas dan semua $R$-modul adalah $R$-modul unital. Barisan $0 \rightarrow A \stackrel{f}{\rightarrow} B \stackrel{g}{\rightarrow} C \rightarrow 0$ disebut barisan eksak pendek jika $\operatorname{Im} f=\operatorname{ker}(g)$. Barisan $0 \rightarrow \mathbb{Z}_{p} \stackrel{f}{\rightarrow} \mathbb{Z}_{p q} \stackrel{g}{\rightarrow} \mathbb{Z}_{q} \rightarrow 0$ merupakan salah satu contoh barisan eksak pendek. Secara umum barisan $R$-modul dan $R$-homomorfisma $\cdots$ $\rightarrow M_{i-1} \stackrel{f_{i}}{\rightarrow} M_{i} \stackrel{f_{i+1}}{\rightarrow} M_{i+1} \longrightarrow \cdots$ disebut eksak di $M_{i}$ jika $\operatorname{Im}\left(f_{i}\right)=\operatorname{ker}\left(f_{i+1}\right)$ atau $\operatorname{Im}\left(f_{i}\right)=$ $f_{i+1}^{-1}(\{0\})$. Apabila sebarang submodul $U_{i-1}$ dari $M_{i-1}$ disubstitusikan pada submodul $\{0\}$, Davvaz dan Parnian- Garamaleky pada tahun 1999 memperkenalkan konsep dari barisan $U$-eksak yang memperumum definsi barisan eksak. Konsep barisan $U$-eksak banyak digunakan secara luas dalam Teori Ring dan Modul, Teori Grup, Teori Homologi, Aljabar Topologi, dan Teori Kompleks.

Barisan $R$-modul dan $R$-homomorfisma $\cdots \rightarrow M_{i-1} \stackrel{f_{i}}{\rightarrow} M_{i} \stackrel{f_{i+1}}{\longrightarrow} M_{i+1} \rightarrow \cdots \quad$ disebut $U_{i+1}$-eksak di $M_{i}$ jika $\operatorname{Im}\left(f_{i}\right)=f_{i+1}^{-1}\left(U_{i+1}\right)$. Dari definisi ini diperoleh bahwa barisan eksak pendek $0 \rightarrow A \stackrel{f}{\rightarrow} B \stackrel{g}{\rightarrow} C \rightarrow 0$ merupakan barisan $\{0\}$-eksak di $A, U$-eksak di $B$, dan $\{0\}$-eksak di $C$, atau sederhananya disebut $U$-eksak. Akibat lain yang diperoleh dari definisi barisan $U$-eksak adalah barisan $0 \rightarrow A \stackrel{f}{\rightarrow} B \stackrel{g}{\rightarrow} C \rightarrow 0$ adalah $U$-eksak jika dan hanya jika $f$ injektif, $g$ surjektif, dan $\operatorname{Im}(f)=$ $g^{-1}(U)$. Misalnya barisan $0 \rightarrow 2 \mathbb{Z} \rightarrow \mathbb{Z} \rightarrow \mathbb{Z}_{4} \rightarrow 0$ merupakan barisan $\mathbb{Z}_{4}$-eksak.

Jika $M$ adalah $R$-modul dan $U$ adalah submodul dari $M$, maka barisan $0 \rightarrow U \stackrel{\subseteq}{\rightarrow} M$ $\stackrel{i}{\rightarrow} M \rightarrow 0$ adalah barisan $U$-eksak. Selanjutnya, jika
$U$ dan $V$ adalah submodul-submodul dari $M$ sedemikian hingga $V \subseteq U \subseteq M$, maka barisan $0 \rightarrow$ $U \stackrel{\subseteq}{\rightarrow} M \stackrel{\pi}{\rightarrow} M / V \rightarrow 0$ adalah barisan $U / V$-eksak dimana $\pi$ adalah homomorfisma natural. Barisan $U$-eksak $0 \rightarrow A \stackrel{f}{\rightarrow} B \stackrel{g}{\rightarrow} C \rightarrow 0$ disebut eksak jika dan hanya jika $U=\{0\}$ sehingga barisan $U$-eksak merupakan perumuman dari barisan eksak.

Dalam tulisan ini diawali dengan definisi dan karakterisasi dari barisan $U$-eksak. Selanjutnya dikaji perumuman Lemma Snake dan Lemma Lima yang memanfaatkan karakterisasi dari barisan $U$ eksak. Karakterisasi dari barisan $U$-eksak diambil dari [2], pembuktian Lemma Snake dan Lemma Lima dapat dilihat di [1] dan [5], sedangkan perumuman Lemma Snake dan Lemma Lima merujuk pada [3] dan [4].

\section{PEMBAHASAN}

Definisi 1. Barisan dari dari $R$-modul dan $R$ homomorfisma

$$
\cdots \rightarrow M_{i-1} \stackrel{f_{i}}{\rightarrow} M_{i} \stackrel{f_{i+1}}{\rightarrow} M_{i+1} \longrightarrow \cdots
$$

disebut barisan $U_{i+1}$-eksak di $M_{i}$ jika $\operatorname{Im}\left(f_{i}\right)=$ $f_{i+1}^{-1}\left(U_{i+1}\right)$, dimana $U_{i+1}$ adalah submodul dari $M_{i+1}$.

Definisi 2. Barisan $U$-eksak $0 \rightarrow A \stackrel{f}{\rightarrow} B \stackrel{g}{\rightarrow} C \rightarrow 0$ isomorfis dengan barisan $U^{\prime}$-eksak $0 \rightarrow A^{\prime} \stackrel{f^{\prime}}{\rightarrow} B^{\prime}$ $\stackrel{g^{\prime}}{\rightarrow} C^{\prime} \rightarrow 0$ jika terdapat diagram komutatif dari $R$ homomorfisma:

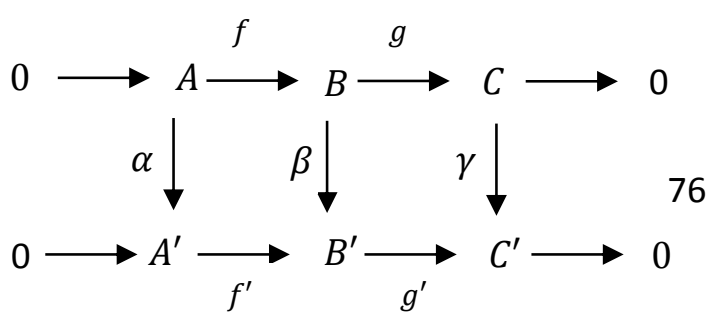


sedemikian hingga $\alpha, \quad \beta$, dan $\gamma$ adalah isomorfisma.

Teorema 3. Isomorfisma dari barisan $U$-eksak merupakan relasi ekuivalen.

Bukti. Harus ditunjukkan isomorfisma dari barisan $U$-eksak dan $U^{\prime}$-eksak merupakan relasi refleksif, simetris, dan transitif. Dengan mengambil $\alpha, \beta$, dan $\gamma$ berupa pemetaan identitas, jelas $\alpha, \beta$, dan $\gamma$ merupakan ismorfisma. Sehingga diperoleh ismorfisma dari barisan $U$-eksak memenuhi sifat refleksif. Selanjutnya, misalkan barisan $U$-eksak $0 \rightarrow A \stackrel{f}{\rightarrow} B \stackrel{g}{\rightarrow} C \rightarrow 0$ isomorfis dengan barisan $U^{\prime}$ eksak $\quad 0 \rightarrow A^{\prime} \stackrel{f^{\prime}}{\rightarrow} B^{\prime} \stackrel{g^{\prime}}{\rightarrow} C^{\prime} \rightarrow 0$, maka terdapat diagram komutatif

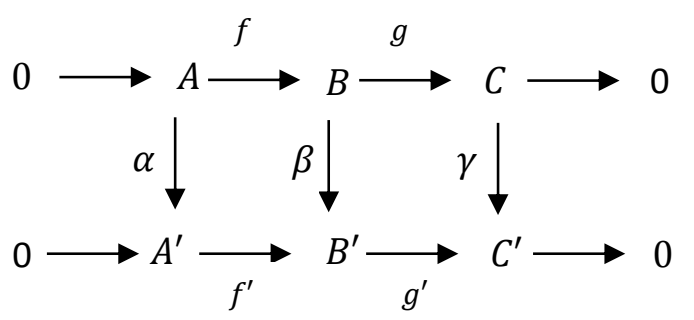

sedemikian hingga $\alpha, \quad \beta$, dan $\gamma$ adalah isomorfisma. Karena $\alpha, \beta$, dan $\gamma$ adalah isomorfisma, maka terdapat $\alpha^{-1}, \beta^{-1}$, dan $\gamma^{-1}$ yang juga adalah isomorfisma. Sehingga barisan $U^{\prime}$ eksak $0 \rightarrow A^{\prime} \stackrel{f^{\prime}}{\rightarrow} B^{\prime} \stackrel{g^{\prime}}{\rightarrow} C^{\prime} \rightarrow 0$ isomorfis dengan barisan $\quad U$-eksak $\quad 0 \rightarrow A \stackrel{f}{\rightarrow} B \stackrel{g}{\rightarrow} C \rightarrow 0$ atau ismorfisma dari barisan $U$-eksak memenuhi sifat simetris. Tinggal ditunjukkan ismorfisma dari barisan $U$-eksak memenuhi sifat transitif. Misalkan misalkan barisan $\quad U$-eksak $0 \rightarrow A \stackrel{f}{\rightarrow} B \stackrel{g}{\rightarrow} C \rightarrow 0$ isomorfis dengan barisan $U^{\prime}$-eksak $0 \rightarrow A^{\prime} \stackrel{f^{\prime}}{\rightarrow} B^{\prime}$ $\stackrel{g^{\prime}}{\rightarrow} C^{\prime} \rightarrow 0 \quad$ dan barisan $\quad U^{\prime}$-eksak $\quad 0 \rightarrow A^{\prime} \stackrel{f^{\prime}}{\rightarrow} B^{\prime}$ $\stackrel{g^{\prime}}{\rightarrow} C^{\prime} \rightarrow 0$ isomorfis dengan barisan $U^{\prime \prime}$-eksak $0 \rightarrow$ $A^{\prime \prime} \stackrel{f^{\prime \prime}}{\rightarrow} B^{\prime \prime} \stackrel{g^{\prime \prime}}{\rightarrow} C^{\prime \prime} \rightarrow 0$ sehingga terdapat diagram komutatif

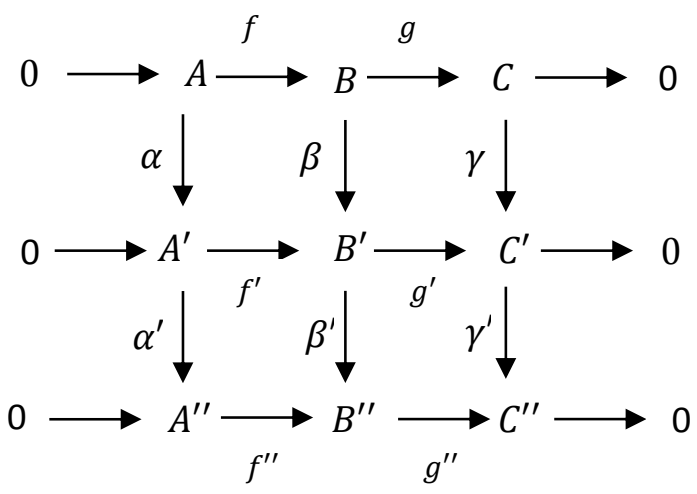

sedemikian hingga $\alpha, \beta, \gamma, \alpha^{\prime}, \beta^{\prime}$, dan $\gamma^{\prime}$ adalah isomorfisma. Dengan memilih $\alpha \circ \alpha^{\prime}, \beta \circ \beta^{\prime}$, dan $\gamma \circ \gamma^{\prime}$ yang juga merupakan isomorfisma, diperoleh barisan $U$-eksak $0 \rightarrow A \stackrel{f}{\rightarrow} B \stackrel{g}{\rightarrow} C \rightarrow 0$ isomorfis dengan barisan $U^{\prime \prime}$-eksak $0 \rightarrow A^{\prime \prime} \stackrel{f^{\prime \prime}}{\rightarrow} B^{\prime \prime} \stackrel{g^{\prime \prime}}{\rightarrow} C^{\prime \prime} \rightarrow$ 0 . Sehingga ismorfisma dari barisan $U$-eksak memenuhi sifat transitif.

Teorema 4. Jika barisan $U$-eksak dan $U^{\prime}$-eksak isomorfis, maka $U \cong U^{\prime}$.

Bukti. Diberikan barisan $U$-eksak dan $U^{\prime}$-eksak isomorfis, maka terdapat diagram komutatif

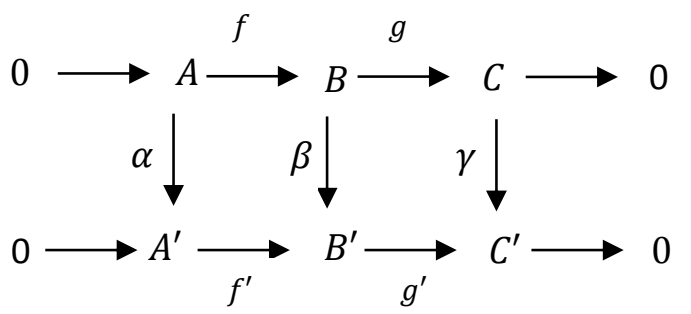

sedemikian hingga $\alpha, \quad \beta$, dan $\gamma$ adalah isomorfisma. Untuk menunjukkan $U \cong U^{\prime}$, cukup ditunjukkan $\gamma(U)=U^{\prime}$. Ambil sebarang $x \in \gamma(U)$, sehingga terdapat $u \in U$ sehingga $x=\gamma(u)$. Karena $u \in U$ dan baris pertama merupakan barisan $U$-eksak, maka $g^{-1}(u) \subseteq g^{-1}(U)=\operatorname{Im} f$ sehingga $g g^{-1}(u) \subseteq g f(A)$. Akibatnya, $u \in$ $g f(A)$, yaitu terdapat $a_{0} \in A$ sedemikian hingga $u=g f\left(a_{0}\right)$. Perhatikan bahwa,

$$
\begin{gathered}
x \quad=\gamma(u)=\gamma\left(g f\left(a_{0}\right)\right)=(\gamma g) f\left(a_{0}\right)= \\
\left(g^{\prime} \beta\right) f\left(a_{0}\right)=g^{\prime}(\beta f)\left(a_{0}\right) \\
=g^{\prime}\left(f^{\prime} \alpha\right)\left(a_{0}\right)=\left(g^{\prime} f^{\prime}\right)\left(\alpha\left(a_{0}\right)\right)
\end{gathered}
$$

Akibatnya, $x \in g^{\prime} f^{\prime}\left(A^{\prime}\right) \subseteq U^{\prime}$ atau $\gamma(U) \subseteq U^{\prime}$. Selanjutnya, ambil $y \in U^{\prime}$. Karena $g^{\prime}$ epimorfisma, maka terdapat $b \in B^{\prime}$ sehingga $g^{\prime}(b)=y$ atau $b=g^{\prime-1}(y)$. Mengingat baris kedua merupakan barisan $U^{\prime}$-eksak, yaitu $\operatorname{Im} f^{\prime}=g^{\prime-1}\left(U^{\prime}\right)$, maka terdapat $a \in A$ sehingga $b=f^{\prime}(a)$. Karena $\gamma$ dan $\beta$ adalah isomorfisma, maka $y=g^{\prime}(b)=$ $g^{\prime} f^{\prime}(a)=g^{\prime} \gamma^{-1} g^{\prime}\left(f^{\prime}(a)\right)=$. Sehingga $y=$ $\gamma\left(g \beta^{-1}(b)\right)=(\gamma g)\left(\beta^{-1}(b)\right)=g^{\prime} \beta\left(\beta^{-1}(b)\right)=$ $g^{\prime}(b)$.

Lemma 5 (Lemma Lima). Diberikan diagram komutatif dari $R$-modul homomorfisma berikut dimana setiap baris merupakan barisan eksak:

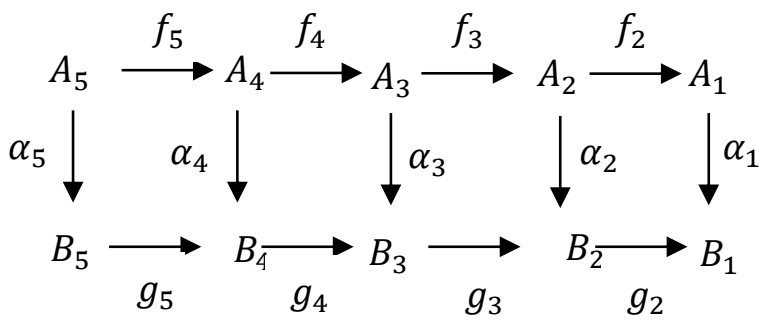


(ii) Akan ditunjukkan $\alpha_{3}$ adalah epimorfisma. Ambil sebarang $x \in B_{3}$, maka dimiliki $g_{3}(x) \in B_{2}$. Karena $\alpha_{2}$ epimorfisma, maka terdapat $y \in A_{2}$ sehingga $\alpha_{2}(y)=g_{3}(x)$. Mengingat baris kedua $\{0\}$-eksak di $B_{2}$, diperoleh $\quad 0=g_{2} g_{3}(x)=g_{2} \alpha_{2}(y)=$ $\alpha_{1} f_{2}(y)$. Sehingga, $f_{2}(y) \in \operatorname{Ker} \alpha_{1}=0$. Akibatnya, $y \in \operatorname{Ker} f_{2} \subseteq f_{2}{ }^{-1}(U)=\operatorname{Im} f_{3}$, yaitu terdapat $z \in A_{3}$ sedemikian hingga $f_{3}(z)=y$. Selanjutnya, $\alpha_{2}(y)=\alpha_{2} f_{3}(z)=$ $g_{3} \alpha_{3}(z)=g_{3}(x)$ karena $\alpha_{2}(y)=g_{3}(x)$. Sehingga, $\quad g_{3}\left(\alpha_{3}(z)-x\right)=0 \quad$ atau $\alpha_{3}(z)-x \in \operatorname{Ker} g_{3} \subseteq g_{3}{ }^{-1}\left(U^{\prime}\right)=\operatorname{Im} g_{4}$, yaitu terdapat $a \in B_{4}$ sedemikian hingga $\alpha_{3}(z)-x=g_{4}(a)$. Dilain pihak, $\alpha_{4}$ adalah epimorfisma, yaitu terdapat $b \in A_{4}$ sehingga $\alpha_{4}(b)=a$. Selanjutnya, $g_{4}(a)=$ $g_{4} \alpha_{4}(b)=\alpha_{3} f_{4}(b)=\alpha_{3}(z)-x \quad$ karena $\alpha_{3}(z)-x=g_{4}(a)$. Akibatnya, $\alpha_{3}\left(f_{4}(b)-\right.$ $z)=x$, yaitu $\alpha_{3}$ adalah epimorfisma.

Lemma 6 (Perumuman Lemma Lima). Diberikan diagram komutatif dari $R$-modul homomorfisma berikut dengan baris pertama adalah $U$-eksak di $A_{2}$, $V$-eksak di $A_{3}$ dan $\{0\}$-eksak di $A_{4}$, dan baris kedua adalah $\{0\}$-eksak di $B_{2}, U^{\prime}$-eksak di $B_{3}$ dan $V^{\prime}$-eksak di $B_{4}$ :

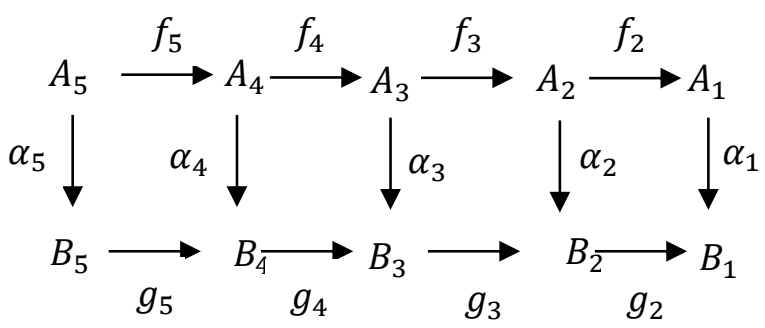

Dengan memanfaatkan (i) dan (ii) diperoleh langsung $\alpha_{3}$ juga isomorfisma.

Lemma 8 (Perumuman Lemma Snake). Misalkan

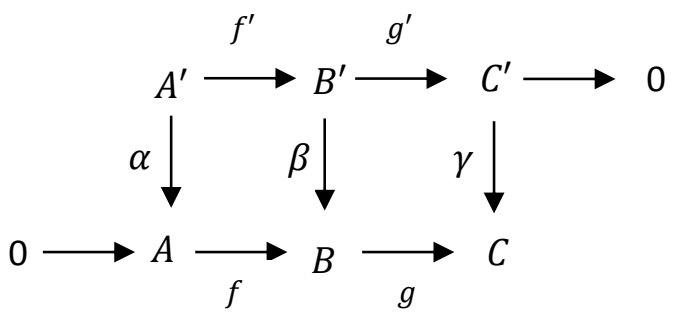

adalah diagram komutatif sedemikian hingga

(1) Baris pertama adalah $U^{\prime}$-eksak dan baris kedua adalah $U$-eksak.

(2) $W \subseteq \operatorname{Im} \alpha$

(3) $U \subseteq \operatorname{Im} \gamma$

(4) $g(V) \subseteq U, f(W)=V$

(5) $\quad U^{\prime} \subseteq \gamma^{-1}(U)$

Maka terdapat homomorfisma $\omega: \frac{\gamma^{-1}(U)}{U^{\prime}} \rightarrow$ Coker $\alpha$ sedemikian hingga barisan : $\alpha_{3}(x)=0$. Karena $\quad 0=g_{3} \alpha_{3}(x)=$ $\alpha_{2} f_{3}(x)$, maka $f_{3}(x) \in \operatorname{Ker} \alpha_{2}=0$. Akibatnya, $x \in \operatorname{Ker} f_{3} \subseteq f_{3}^{-1}(U)=\operatorname{Im} f_{4}$. Sehingga, terdapat $y \in A_{4}$ sedemikian hingga $f_{4}(y)=x$. Perhatikan bahwa : $0=$ $\alpha_{3}(x)=\alpha_{3} f_{4}(y)=g_{4} \alpha_{4}(y)$. Akibatnya, $\alpha_{4}(y) \in \operatorname{Ker} g_{4} \subseteq g_{4}{ }^{-1}\left(U^{\prime}\right)=\operatorname{Im} g_{5}$.

Berarti terdapat $z \in B_{5}$ sehingga $g_{5}(z)=$ $\alpha_{4}(y)$. Mengingat $\alpha_{5}$ epimorfisma, maka terdapat $w \in A_{5} \quad$ sehingga $\quad \alpha_{5}(w)=z$. Selanjutnya, $\quad \alpha_{4}(y)=g_{5}(z)=g_{5} \alpha_{5}(w)=$ $\alpha_{4} f_{5}(w)$ atau $\alpha_{4}\left(y-f_{5}(w)\right)=0$. Karena $\alpha_{4}$ monomorfisma, maka $y-f_{5}(w)=0$ atau $y=f_{5}(w)$. Dengan memanfaatkan $\{0\}$-eksak pada $A_{4}$ diperoleh $x=f_{4}(y)=$ $f_{4} f_{5}(w)=0$. Dengan demikian $\alpha_{3}$ adalah monomorfisma.

$$
\begin{aligned}
& \alpha^{-1}(W) \stackrel{f_{*}^{\prime}}{\rightarrow} \beta^{-1}(V) \stackrel{g_{*}^{\prime}}{\rightarrow} \frac{\gamma^{-1}(U)}{U^{\prime}} \stackrel{\omega}{\rightarrow} \text { Coker } \alpha \\
& \stackrel{f_{*}^{\prime}}{\rightarrow} \text { Coker } \beta \stackrel{g_{*}^{\prime}}{\rightarrow} \text { Coker } \gamma
\end{aligned}
$$

eksak.

Bukti. Ambil $x \in g^{\prime}\left(\beta^{-1}(V)\right)$, maka terdapat $y \in$ $V$ sedemikian hingga $g^{\prime}\left(\beta^{-1}(y)\right)=x$. Karena $y \in$ $V$ maka $g(y) \in C$, dan $g(V) \subseteq U$ mengakibatkan $g(y) \in U . \quad$ Sehingga $\quad \gamma^{-1}(g(y)) \in \gamma^{-1}(U)$. Akibatnya $\quad g^{\prime}\left(\beta^{-1}(y)\right)=\gamma^{-1}(g(y)) \in \gamma^{-1}(U)$. Sehingga $g^{\prime}\left(\beta^{-1}(V)\right) \subseteq \gamma^{-1}(U)$. Dengan cara yang sama dapat ditunjukkan $f^{\prime}\left(\alpha^{-1}(W)\right) \subseteq \beta^{-1}(V)$. Akibatnya terbentuk barisan:

$$
\alpha^{-1}(W) \stackrel{\left.f^{\prime}\right|_{\alpha^{-1}(W)}}{\longrightarrow} \beta^{-1}(V) \stackrel{\left.g^{\prime}\right|_{\beta^{-1}(V)}}{\longrightarrow} \gamma^{-1}(U)
$$


Dibentuk homomorfisma kanonik $\pi: \gamma^{-1}(U) \rightarrow$ $\frac{\gamma^{-1}(U)}{U \prime}$. Misalkan $f_{*}^{\prime}=\left.f^{\prime}\right|_{\alpha^{-1}(W)}$ dan $g_{*}^{\prime}=$ $\left.\pi g^{\prime}\right|_{\beta^{-1}(V)}$ sehingga diperoleh barisan:

(I)

$$
\stackrel{f_{*}^{\prime}}{\rightarrow} \beta^{-1}(V) \stackrel{g_{*}^{\prime}}{\rightarrow} \frac{\gamma^{-1}(U)}{U^{\prime}}
$$

Dilain pihak, dari $f$ dan $g$ dapat dibentuk homomorfisma $f^{*}: \frac{A}{\operatorname{Im} \alpha} \rightarrow \frac{B}{\operatorname{Im} \beta}$ dengan $f^{*}(a+$ $\operatorname{Im} \alpha)=f(a)+\operatorname{Im} \beta, \quad$ dan $\quad g^{*}: \frac{B}{\operatorname{Im} \beta} \rightarrow \frac{C}{\operatorname{Im} \gamma}$ dengan $g^{*}(b+\operatorname{Im} \beta)=g(b)+\operatorname{Im} \gamma$. Sehingga diperoleh barisan:

(II)

\section{Coker $\alpha \stackrel{f^{*}}{\rightarrow}$ Coker $\beta \stackrel{g^{*}}{\rightarrow}$ Coker $\gamma$}

Selanjutnya akan ditunjukkan terdapatnya homomorfisma $\omega: \frac{\gamma^{-1}(U)}{U^{\prime}} \rightarrow$ Coker $\alpha$ yang menghubungkan barisan (I) dan (II). Misalkan $z+$ $U^{\prime} \in \frac{\gamma^{-1}(U)}{U^{\prime}}$. Pilih $b^{\prime} \in B^{\prime}$ sehingga $g^{\prime}\left(b^{\prime}\right)=z$. Karena $g \beta\left(b^{\prime}\right)=\gamma g^{\prime}\left(b^{\prime}\right)=\gamma(z) \in U, \quad$ maka $\beta\left(b^{\prime}\right) \in g^{-1}(U)$. Mengingat barisan (II) eksak, maka $\beta\left(b^{\prime}\right) \in \operatorname{Im} f$. Akibatnya terdapat dengan tunggal $a \in A$ sedemikian hingga $\beta\left(b^{\prime}\right)=f(a)$ dan $a=f^{-1} \beta\left(b^{\prime}\right)$. Didefinisikan $\omega\left(z+U^{\prime}\right)=a+$ Im $\alpha$. Misalkan $b^{\prime \prime} \in B^{\prime}$ dengan $g^{\prime}\left(b^{\prime \prime}\right)=z$, maka terdapat $a^{\prime} \in A$ sedemikian hingga $\beta\left(b^{\prime \prime}\right)=f\left(a^{\prime}\right)$. Dari $g^{\prime}\left(b^{\prime}\right)=z$ dan $g^{\prime}\left(b^{\prime \prime}\right)=z$, diperoleh $g^{\prime}\left(b^{\prime}-\right.$ $\left.b^{\prime \prime}\right)=0 . \quad$ Akibatnya $\quad b^{\prime}-b^{\prime \prime} \in \operatorname{Ker} g^{\prime} \subseteq$ $g^{\prime-1}\left(U^{\prime}\right)=\operatorname{Im} f^{\prime}$, sehingga terdapat $\bar{a} \in A^{\prime}$ dengan $b^{\prime}-b^{\prime \prime}=f^{\prime}(\bar{a}) . \quad$ Karena $\quad \beta f^{\prime}(\bar{a})=f \alpha(\bar{a})$, diperoleh $\beta\left(b-b^{\prime \prime}\right)=f \alpha(\bar{a})$ yang berakibat $f\left(a-a^{\prime}\right)=f \alpha(\bar{a})$. Mengingat $f$ monomorfisma, maka $a-a^{\prime}=\alpha(\bar{a}) \in \operatorname{Im} \alpha$. Akibatnya $a+$ $\operatorname{Im} \alpha=a^{\prime}+\operatorname{Im} \alpha$, yaitu $\omega$ terdefinisi dengan baik. Tinggal ditunjukkan keeksakan

$$
\begin{aligned}
& \alpha^{-1}(W) \stackrel{f_{*}^{\prime}}{\rightarrow} \beta^{-1}(V) \stackrel{g_{*}^{\prime}}{\rightarrow} \frac{\gamma^{-1}(U)}{U^{\prime}} \stackrel{\omega}{\rightarrow} \text { Coker } \alpha \\
& \stackrel{f_{*}^{\prime}}{\rightarrow} \text { Coker } \beta \stackrel{g_{*}^{\prime}}{\rightarrow} \text { Coker } \gamma
\end{aligned}
$$

Langkah 1 : Akan ditunjukkan $\operatorname{Im} f_{*}^{\prime}=\operatorname{Ker} g_{*}^{\prime}$.

Ambil $\quad b^{\prime} \in \operatorname{Ker} g_{*}^{\prime}$. Berarti $g_{*}^{\prime}\left(b^{\prime}\right)=0+U^{\prime}$ sehingga diperoleh $g^{\prime}\left(b^{\prime}\right) \in U^{\prime}$. Akibatnya $b^{\prime} \in$ $g^{\prime-1}\left(U^{\prime}\right)$. Karena baris (I) eksak, terdapat $x \in A^{\prime}$ sehingga $\quad b^{\prime}=f^{\prime}(x)$. Karena $f_{*}^{\prime}=\left.f^{\prime}\right|_{\alpha^{-1}(W)}$, tinggal ditunjukkan $x \in \alpha^{-1}(W)$. Perhatikan bahwa: $f \alpha(x)=\beta f^{\prime}(x)=\beta\left(b^{\prime}\right)$. Karena $b^{\prime} \in$ $\beta^{-1}(V)$, maka $\beta\left(b^{\prime}\right) \in V$. Akibatnya $f \alpha(x) \in V$. Dilain pihak $f(W)=V$ dan $f$ monomorfisma, maka $\alpha(x) \in W$ atau $x \in \alpha^{-1}(W)$. Akibatnya Ker $g_{*}^{\prime} \subseteq$ $\operatorname{Im} f_{*}^{\prime}$. Selanjutnya, ambil $a \in \operatorname{Im} f_{*}^{\prime}$ berarti terdapat $x \in \alpha^{-1}(W)$ sehingga $f_{*}^{\prime}(x)=a$ atau $\left.f^{\prime}\right|_{\alpha^{-1}(W)}=$ $a, f^{\prime}(x)=a$. Akibatnya $a \in \operatorname{Im} f^{\prime}=g^{\prime-1}\left(U^{\prime}\right)$, yaitu $g^{\prime}(a) \in U^{\prime}$. Sehingga $\operatorname{Im} f_{*}^{\prime} \subseteq \operatorname{Ker} g_{*}^{\prime}$, dengan kata lain $\operatorname{Im} f_{*}^{\prime}=\operatorname{Ker} g_{*}^{\prime}$.

Langkah 2: $\operatorname{Im} g_{*}^{\prime}=\operatorname{Ker} \omega$.
Ambil $x \in I m g_{*}^{\prime}$ terdapat $b^{\prime} \in \beta^{-1}(V)$ sehingga $g^{\prime}\left(b^{\prime}\right)+U^{\prime}=x$. Selanjutnya $\omega(x)=\omega\left(g^{\prime}\left(b^{\prime}\right)+\right.$ $\left.U^{\prime}\right)=f^{-1} \beta\left(g^{\prime-1}\left(g^{\prime}\left(b^{\prime}\right)\right)\right)+\operatorname{Im} \alpha=f^{-1} \beta\left(b^{\prime}\right)+$ Im $\alpha$. Karena $b^{\prime} \in \beta^{-1}(V)$ maka $\beta\left(b^{\prime}\right) \in V$. Mengingat $f(W)=V$ dan $f$ monomorfisma maka $f^{-1} \beta\left(b^{\prime}\right) \in W \subseteq \operatorname{Im} \alpha$. Akibatnya $\omega(x) \in \operatorname{Im} \alpha$ sehingga $x \in \operatorname{Ker} \omega$. Jadi $\operatorname{Im} g_{*}^{\prime} \subseteq \operatorname{Ker} \omega$. Ambil $y=c^{\prime}+U^{\prime} \in \operatorname{Ker} \omega$ dengan $c^{\prime} \in \gamma^{-1}(U)$ berarti $\omega\left(c^{\prime}+U^{\prime}\right)=\operatorname{Im} \alpha \quad$ atau $\quad f^{-1} \beta\left(g^{\prime-1}\left(c^{\prime}\right)\right)+$ $\operatorname{Im} \alpha \in \operatorname{Im} \alpha$. Sehingga terdapat $x \in A^{\prime}$ sedemikian hingga $\beta\left(g^{\prime-1}\left(c^{\prime}\right)\right)=f \alpha(x)=\beta f^{\prime}(x)$.

Akibatnya $\quad \beta\left(g^{\prime-1}\left(c^{\prime}\right)-f^{\prime}(x)\right)=0 \quad$ yaitu $g^{\prime-1}\left(c^{\prime}\right)-f^{\prime}(x) \in \operatorname{Ker} \beta \subseteq \beta^{-1}(V)$. Sehingga $g_{*}^{\prime}\left(g^{\prime^{-1}}\left(c^{\prime}\right)-f^{\prime}(x)\right)=\pi g^{\prime}\left(g^{\prime-1}\left(c^{\prime}\right)-\right.$ $\left.f^{\prime}(x)\right)=g^{\prime}\left(g^{\prime-1}\left(c^{\prime}\right)-f^{\prime}(x)\right)+U^{\prime}=c^{\prime}+U^{\prime}=$ $y$. Akibatnya Ker $\omega \subseteq \operatorname{Im} g_{*}^{\prime}$. Dengan demikian $\operatorname{Im} g_{*}^{\prime}=\operatorname{Ker} \omega$.

Langkah $3: \operatorname{Im} \omega=\operatorname{Ker} f^{*}$.

Ambil $x \in \operatorname{Im} \omega$ yaitu terdapat $c^{\prime}+U^{\prime} \in \frac{\gamma^{-1}(U)}{U^{\prime}}$ sehingga $\omega\left(c^{\prime}+U^{\prime}\right)=x$. Perhatikan bahwa: $f^{-1} \beta\left(g^{\prime-1}\left(c^{\prime}\right)\right)+\operatorname{Im} \alpha=x$,

$f^{*}\left[f^{-1} \beta\left(g^{\prime-1}\left(c^{\prime}\right)\right)+\operatorname{Im} \alpha\right]=f^{*}[x]$,

$\beta\left(g^{\prime-1}\left(c^{\prime}\right)\right)+\operatorname{Im} \beta=f^{*}(x)$. Sehingga $x \in$ Ker $f^{*}$ atau $\operatorname{Im} \omega \subseteq \operatorname{Ker} f^{*}$. Selanjutnya misalkan $y=$ $a+\operatorname{Im} \alpha \in \operatorname{Ker} f^{*}$ berarti $f^{*}(a+\operatorname{Im} \alpha)=\operatorname{Im} \beta$ atau $f(a)+\operatorname{Im} \beta=\operatorname{Im} \beta$, sehingga $f(a) \in \operatorname{Im} \beta$. Akibatnya terdapat $b^{\prime} \in B^{\prime}$ sedemikian hingga $\beta\left(b^{\prime}\right)=f(a)$. Mengingat $g \beta=\gamma g^{\prime}$ diperoleh $\gamma g^{\prime}\left(b^{\prime}\right) \in U$. Akibatnya, $g^{\prime}\left(b^{\prime}\right) \in \gamma^{-1}(U)$ sehingga $\omega\left(g^{\prime}\left(b^{\prime}\right)+U^{\prime}\right)=f^{-1} \beta\left(b^{\prime}\right)+\operatorname{Im} \alpha=a+$

$\operatorname{Im} \alpha=y$. Diperoleh Ker $f^{*} \subseteq \operatorname{Im} \omega$ sehingga Ker $f^{*}=\operatorname{Im} \omega$.

Langkah $4: \operatorname{Im} f^{*}=\operatorname{Ker} g^{*}$.

Misalkan $f^{*}(a+\operatorname{Im} \alpha) \in \operatorname{Im} f^{*}$, maka $g^{*} f^{*}(a+$ $\operatorname{Im} \alpha)=g f(a)+\operatorname{Im} \gamma$. Karena $g f(a) \in U \subseteq$ $\operatorname{Im} \gamma$ maka $g^{*} f^{*}(a+\operatorname{Im} \alpha) \in \operatorname{Im} \gamma$. Akibatnya $f^{*}(a+\operatorname{Im} \alpha) \in \operatorname{Ker} g^{*}, \quad$ yaitu $\operatorname{Im} f^{*} \subseteq \operatorname{Ker} g^{*}$. Selanjutnya ambil $b+\operatorname{Im} \beta \in \operatorname{Ker} g^{*}$, berarti $g^{*}(b+\operatorname{Im} \beta)=g(b)+\operatorname{Im} \gamma \in \operatorname{Im} \gamma$. Sehingga terdapat $c^{\prime} \in C^{\prime}$ sedemikian hingga $g(b)=\gamma\left(c^{\prime}\right)$. Karena $g^{\prime}$ epimorfisma, maka $g(b)=\gamma g^{\prime}\left(b^{\prime}\right)=$ $g \beta\left(b^{\prime}\right)$. Ini berakibat $g\left(b-\beta\left(b^{\prime}\right)\right)=0$ atau $b-$ $\beta\left(b^{\prime}\right) \in \operatorname{Ker} g \subseteq g^{-1}(U)=\operatorname{Im} f$. Sehingga terdapat $a \in A$ sedemikian hingga $b+\operatorname{Im} \beta=$ $f(a)+\operatorname{Im} \beta=f^{*}(a+\operatorname{Im} \alpha)$, yaitu Ker $g^{*} \subseteq \operatorname{Im} f^{*}$. Dengan demikian $\operatorname{Im} f^{*}=\operatorname{Ker} g^{*}$.

Akibat 9. Misalkan

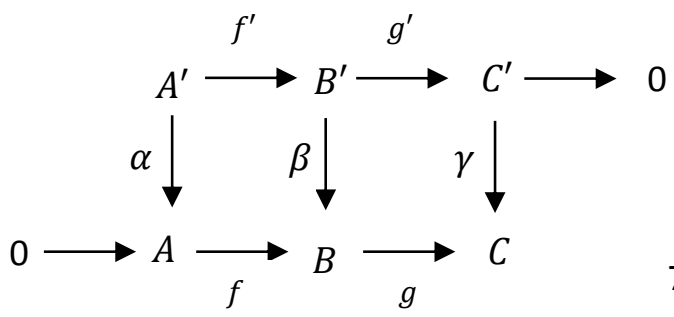


adalah diagram komutatif sedemikian hingga

(1) Baris pertama adalah $U^{\prime}$-eksak dan baris kedua adalah $U$-eksak.

(2) $U^{\prime} \subseteq \gamma^{-1}(U)$ dan $U \subseteq \operatorname{Im} \gamma$.

Maka barisan :

$$
\begin{aligned}
& \operatorname{Ker} \alpha \stackrel{f_{*}^{\prime}}{\rightarrow} \operatorname{Ker} \beta \stackrel{g_{*}^{\prime}}{\rightarrow} \frac{\gamma^{-1}(U)}{U^{\prime}} \stackrel{\omega}{\rightarrow} \text { Coker } \alpha \\
& \stackrel{f_{*}^{\prime}}{\rightarrow} \text { Coker } \beta \stackrel{g_{*}^{\prime}}{\rightarrow} \text { Coker } \gamma
\end{aligned}
$$

eksak.

\section{DAFTAR PUSTAKA}

[1] Anderson, F.W. and Fuller, K.R., Rings and Categories of Modules, Springer-Verlag, New York, Inc. 1992.

[2] Davvaz, B. and Parnian-Garamaleky, Y.A., A Note On Exact Sequences, Bull. Malaysian Math. Soc. 22 (1999), No. 2, pp. 53-56.

[3] Davvaz, B. and Shabani-Solt, H., A Generalization Of Homological Algebra, J. Korean Math. Soc. 39 (2002), No. 6, pp. 881-898.

[4] Madanshekaf, A., Quasi-Exact Sequence and Finitely Presented Modules, Iranian Journal of Mathematical Sciences and Informatics, Vol. 3 (2008), No. 2, pp. 4953.

[5] Wisbauer, R., Foundations of Module and Ring Theory, Gordon and Breach : Philade, 1991. 